\title{
Preconditioning by the inhalation of pure oxygen protects rat's cochlear function against noise-induced hearing loss.
}

\author{
Faridan M, PhD Student ${ }^{1}$, Khavanin A, $\mathrm{PhD}^{2^{*}}$, Mirzaei $\mathrm{R}, \mathrm{PhD}^{3}$ \\ 1- PhD Student in Occupational Health, Dept. of Occupational Health Engineering, Faculty of Medical Sciences, Tarbiat \\ Modares University, Tehran, Iran. 2- Associate Prof., Dept. of Occupational Health Engineering, Faculty of Medical \\ Sciences, Tarbiat Modares University, Tehran, Iran. 3- Professor, Dept. of Environmental and Occupational Health \\ Engineering, School of Health, Mashhad University of Medical Sciences, Mashhad, Iran.
}

\begin{abstract}
Received: October 2017, Accepted: November 2017

Background: Occupational noise-induced hearing loss (ONIHL) is a hearing disorder that affects workers all over the world. Preconditioning with several mild or less potent stressors will effectively prevent the development of noise-induced hearing loss. This study investigated the possible preventive effects of normobaric hyperoxia preconditioning on preventing the noise-induced hearing impairment in the rats.

Materials and Methods: Four groups of male Wistar rats served as controls, exposed to normobaric oxygen, noise, or oxygen plus noise. The noise exposure lasted for 6 hours/day, 5 days/week, 4 weeks and the preconditioning conducted by inhalation of pure oxygen (95\%) for 3 hours/day, 6 days/week, 1 week in a chamber prior to noise exposure. A bandpass noise of 100dB SPL centered at $8 \mathrm{KHz}$ was used for noise exposure. Click and tone burst auditory brainstem responses (ABR) and distortion product otoacoustic emissions (DPOAE) audiograms were recorded in order to evaluate the level of hearing loss among the subjects before the beginning and 4 weeks post-exposure. Statistical analysis was performed using SPSS.

Results: The weekly pre-conditioning by normobaric hyperoxia significantly reduced the ABR threshold shifts and restored the amplitudes of DPOAE $(\mathrm{P}<0.001)$. The control group did not show any difference in terms of DPOAE and ABR tests during and after the exposure $(\mathrm{P}>0.050)$.

Conclusions: The preconditioning successfully protected the rats' hearing by maximizing and developing tolerance to ischemia and hypoxia caused by the noise. The application of such preconditioning is then considered as a possible complementary preventive solution.
\end{abstract}

Keywords: Hyperoxia, Noise-Induced Hearing Loss, Auditory Brainstem Response, Rat

\section{Introduction}

Noise-induced hearing loss (NIHL) is a developing and debilitating disorder usually caused by the damage to the basilar membrane and stereocilia of the outer hair cells (OHCs) of the cochlea in the inner ear.

Globally, NIHL is considered to be a significant health problem and occupational NIHL (ONIHL), as the most prevalent form of NIHL, accounts for a large burden of hearing loss in total. The occupational noise almost leads to $16 \%$ of the severe hearing loss among adults, with estimates of disease burden ranging from $7 \%$ in developed countries to $21 \%$ in underdeveloped and developing nations (1). It is estimated that a considerable amount of burden of hearing loss is caused by ONIHL in the United States (2). In Iran, based on the experts' evaluations, almost 2 million workers are exposed to noise levels exceeding $85 \mathrm{dBA}$, the occupational exposure limit recommended by the Iranian Ministry of Health And Medical Education (3).

The results from the previous studies have suggested that NIHL is either developed by the direct mechanical trauma initiated by the higher levels of the noise or indirect metabolic

* Corresponding author: Ali Khavanin, Dept. of Occupational Health Engineering, Faculty of Medical Sciences, Tarbiat Modares University, Tehran, Iran. E-mail: khavanin@modares.ac.ir 
damage to various components of the organ of Corti (4). Today, almost all researchers agree upon the fact that apart from acoustic trauma cases, the principal exposure to the higher levels of noise will contribute to the developing NIHL through the excessive generation of free radical species such as reactive oxygen species (ROS) and reactive nitrogen species (RNS) within the organ of corti and cochlea which then lead to cell injury or death (5).

In order to minimize the risk of developing ONIHL, occupational hygienists have taken multiple measures such as the modification of noise sources through engineering techniques, applying administrative controls, use of personal hearing protection devices (HPDs) and regular screening tests and training sessions.

Nonetheless, from a practical point of view, such measures are likely hard to be implemented due to multiple challenges; and therefore, alternative or complementary approaches are required. The literature review reveals that several alternative preventive approaches have been suggested to curb the loss of hearing caused by the exposure to high levels of noise and hence the development of NIHL in general (6). Among the welldocumented methods, there are the approaches based on the use of antioxidants to prevent the damage caused by the oxidative stress, the use of blood flow promoting agents to control the level of cochlear hypoxia and approaches based on preconditioning.

There is almost a century of literature that supports the concept that prior injury caused by non-damaging or moderate levels of a variety of stressors (preconditioning) will considerably minimize the effects of subsequent exposures to more harmful or potent stressors. Preconditioning by various stressors such as hypoxia, hyperthermia, restraint, and the noise itself has been reported to be capable of contributing to a moderate or non-injurious level of stress that renders protection against the development of NIHL. Experts believe that the hypothesis underlying such mechanism is likely to be related to the activation of antioxidant enzymes, inhibition of apoptosis, increased tyrosine hydroxylase activity in the lateral efferent system, and glucocorticoid activation (7).

Sub-lethal oxidative stress induced by normobaric hyperoxia (NBHO), an inhalation of almost pure oxygen at normal atmospheric pressure, is one of the preconditioning agents that may enhance several cellular endogenous defense mechanisms and lead to adaptive responses that may in turn induce protection against subsequent stress from the same (tolerance) or other (cross-tolerance) stimuli, mainly through the generation of oxygen-free radicals and hydroxyl radicals (8-11). It is therefore hypothesized that NBHO preconditioning is able to avert the process of free radicals formation in the cochlea caused by exposure to higher levels of the noise.

The present study seeks to determine whether oxygen preconditioning before exposure to noise could protect the hearing and minimize the auditory dysfunction and cochlear damage caused by NIHL.

\section{Material and Methods}

In this experimental-interventional study male Wistar rats (specific-pathogen-free, initial weight approximately 180-200 g) were obtained from Pasteur Institution of Tehran, Iran. They were housed in polypropylene cages $(400 \times 250 \times 150 \mathrm{~mm})$ with steamcleaned pinewood bedding in a controlled animal house at $20-22{ }^{\circ} \mathrm{C}, 40-50 \%$ relative humidity, and 10 times/hour air displacement. The light/dark schedule (12/12 hours) was kept during the exposure period. Apart from the exposure period, food (rodent chow, Pars Animal Feed Co, Iran) and tap water were available ad libitum. All experimental procedures including the care and use of animals in this study were approved by the Ethics in Experimental Medicine Committee of the Tarbiat Modares University, Tehran, Iran, and followed the guidelines of the Declaration of Helsinki (12). 
Table 1: Conditioning process and exposures of experimental groups

\begin{tabular}{cc}
\hline Experimental group & Number of subjects in each group \\
\hline Controls & 6 \\
\hline 100 dBA noise & 6 \\
\hline NBHO (3 hours/day, 1 week) + 100 dBA & 6 \\
\hline NBHO (3 hours/day, 1 week) & 6 \\
\hline NBHO: Normobaric hyperoxia &
\end{tabular}

Rats inhaled the pure normobaric oxygen for 3 hours on a daily basis during a week before their exposure to the noise starts. A bandpass noise centered at $8 \mathrm{KHz}$ and $100 \mathrm{dBA} \pm 1 \mathrm{dBA}$ sound pressure level (SPL) was applied during the exposure which lasted six hours per day five days per week for four consecutive weeks
(6 hours/day, 5 days/week, 4 weeks). The previous studies have shown that such spectrum and level of noise exposure readily induces auditory threshold shifts in the rats (13-15). Table 1 and figure 1 show the details of the condition process and time schedule exposures of experimental groups.

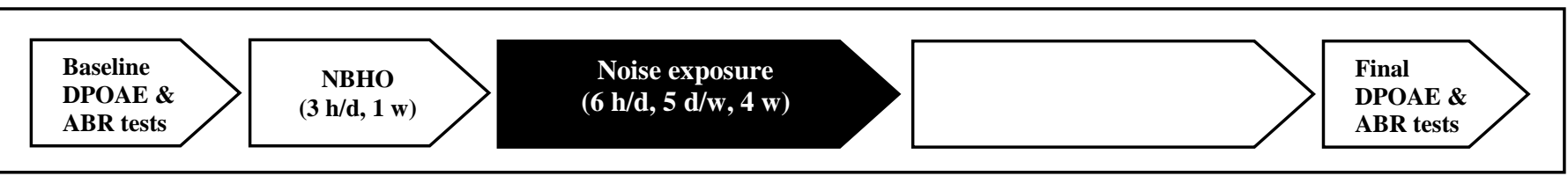

Figure 1: Time schedules of the experimental groups

NBHO: Normobaric hyperoxia; DPOAE: Distortion product otoacoustic emissions; ABR: Auditory brainstem responses

The noise was generated on a computer by a Filtered Noise Generator software (Timo Esser's Audio software, version 1.2), recorded and played by the Cool Edit Pro software (Syntrillium Software Corporation, version 2.1), amplified by an audio amplifier (Pejvak Ava Corporation, Model AP12), delivered by loudspeakers (JBL GT6-6) located approximately $12 \mathrm{~cm}$ above the wire cages and measured at the level of rats' ears in each cage using a sound level meter (Casella CEL 480). Considering the designs proposed by Hinners et al. (16), a custom made reverberant chamber was made of stainless steel and glass in order to expose the animals to the noise. Such design contributed to a dynamic and continuous flow of air inside the chamber. Figure 2A and B depict the pictures of the chambers used in this study.
A

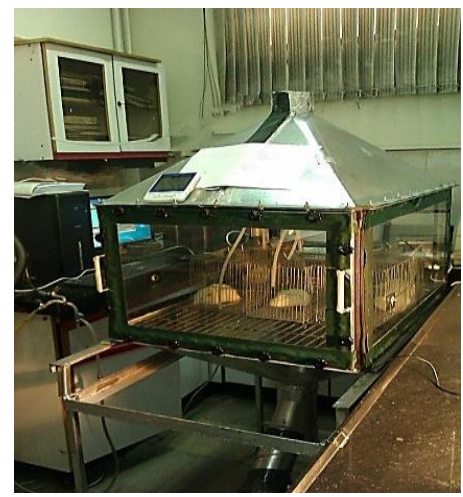

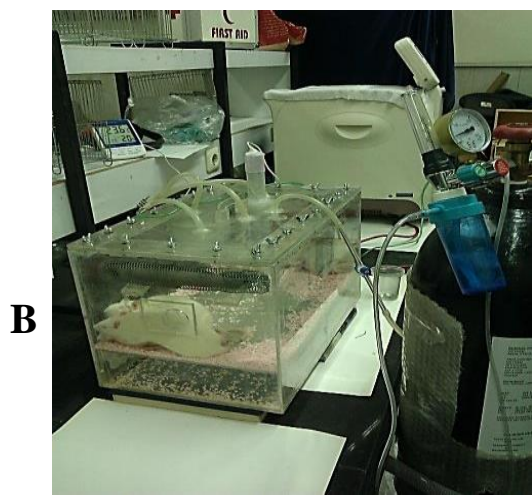

Figure 2: A) The chamber for keeping animals exposed to the noise; B) the chamber for inhaling pure oxygen by the animals. 
The temperature and relative humidity inside the chamber were respectively $21 \pm 1{ }^{\circ} \mathrm{C}$ at 50 $\pm 10 \%$ and monitored by a digital thermometer and humidity meter. Rats were kept individually in stainless steel wire cages $(20 \times$ $20 \times 18$ ) with wire-mesh floors. The cages were positioned symmetrically on a shelf within the noise exposure chamber. The cages in the chamber were daily rotated one position to maintain an equal exposure for all rats.

In order to make animals inhale the oxygen, the animals that received normobaric oxygen (NBHO group) were kept in an airtight Plexiglas chamber $(650 \times 350 \times 450 \mathrm{~mm})$ with gas inflow and outflow outlets for three hours per day, five days per week.

The temperature, humidity, and pressure were continuously monitored by a digital thermometer (SamwonEng model su-105ip), digital hygrometer (Sunward eng model: sun 25-11) and inclined manometer within the chamber and their waste was systematically removed.

The oxygen was supplied by a cylinder containing pure oxygen (99\%) along with an oxygen concentrator (Green Life's) and delivered through a gas flow meter at $\geq 8$ 1/minute. Acrolime (Marlic Medical Ind, Iran) and silica gel (Silica Gel Derakhshan Co, Iran) were used inside the chamber to remove $\mathrm{CO}_{2}$ and humidity. An oxygen meter (Lutron, DO5510) and a $\mathrm{CO}_{2}$ meter (Lutron, GC-2028) continuously measured the level of oxygen inside the chamber.

Distortion product otoacoustic emissions (DPOAE) test and auditory brainstem response (ABR) recordings were employed to assess the animals' auditory function before and after the exposure.

During the ABR and DPOAE tests, the rats were under general anesthesia induced by intraperitoneal (IP) injection of a mixture of ketamine $(50 \mathrm{mg} / \mathrm{kg})$ and xylazine $(5 \mathrm{mg} / \mathrm{kg})$. All measurements were performed while the anesthetized rats were kept inside a small sound-attenuated chamber. The rectal temperatures of these rats were maintained at $38.0{ }^{\circ} \mathrm{C}$ by applying a heating pad that covered their bodies (Deltaphase Isothermal Pads, ASS7T 6" x 7.5").

Based on the previous experiments, DPOAE tests and ABR recordings are mostly stable and have maximum levels at this temperature $(17,18)$. A digital thermometer (VAC-STAR, MH275) was used to ensure that the animals' core temperature was maintained during the experiment. The DPOAE and ABR tests were performed prior to any experimental conditioning (baseline), and 4 weeks postexposure (permanent hearing loss).

DPOAE test was conducted by presenting two pure primary tones $\left(\mathrm{L}_{1}=60\right.$ and $\mathrm{L}_{2}=50 \mathrm{~dB}$ SPL; $\mathrm{L}_{1}-\mathrm{L}_{2}=10 \mathrm{~dB}$ SPL) with $\mathrm{f}_{2} / \mathrm{f}_{1}=1.25$ to measure DPOAE at $f_{2}$ frequencies of $4,6,8$ and $10 \mathrm{kHz}$ as audiograms and signal to noise ratio (SNR) by a DPOAE analyzer (DPOAE 4000 I/O Model, HOMOTH Company, Hamburg, Germany). The inclusion criterion for normal DPOAE amplitude was when between the amplitudes of the obtained curves was $6 \mathrm{~dB}$ above the noise-floor.

ABR thresholds were measured for the left ear of each animal using Biologic Navigator Pro system (Natus, USA) with the intensity level and rate controlled by the program. The thresholds were determined for clicks and pure tones at $4,6,8,12$, and $16 \mathrm{kHz}$ using descending sound level series. Clicks and tone bursts were 5 minutes in duration and presented at a rate of $23.1 /$ second.

Stainless steel needle electrodes (Ambu ${ }^{\circledR}$ NeurolineSubdermal) were inserted subcutaneously at the vertex and the mastoid. A ground electrode was placed in the hind leg. The electrical responses obtained from the electrodes were amplified ( $\times 100,000)$, bandpass filtered $(100-3000 \mathrm{~Hz})$ and averaged at a sample rate of 1024 for each level. The sound stimulus was presented by a tweeter (Brüel \& Kjær), positioned $10 \mathrm{~cm}$ away from the rat's pinna. The ABR threshold was defined as the lowest sound level at which peak II as the reference point could be positively identified by the investigators (19). To confirm response reproducibility, the measurement was performed at least twice. 
Figure 3 shows an ABR recoding from a normal rat.

Shapiro-Wilk test was used for normality test of data. Analysis of variance (ANOVA) and post hoc comparisons (Tukey's test) was performed for data analysis using SPSS software (version 18, SPSS Inc., Chicago, IL, USA). The significance level was consideredas 0.050 .

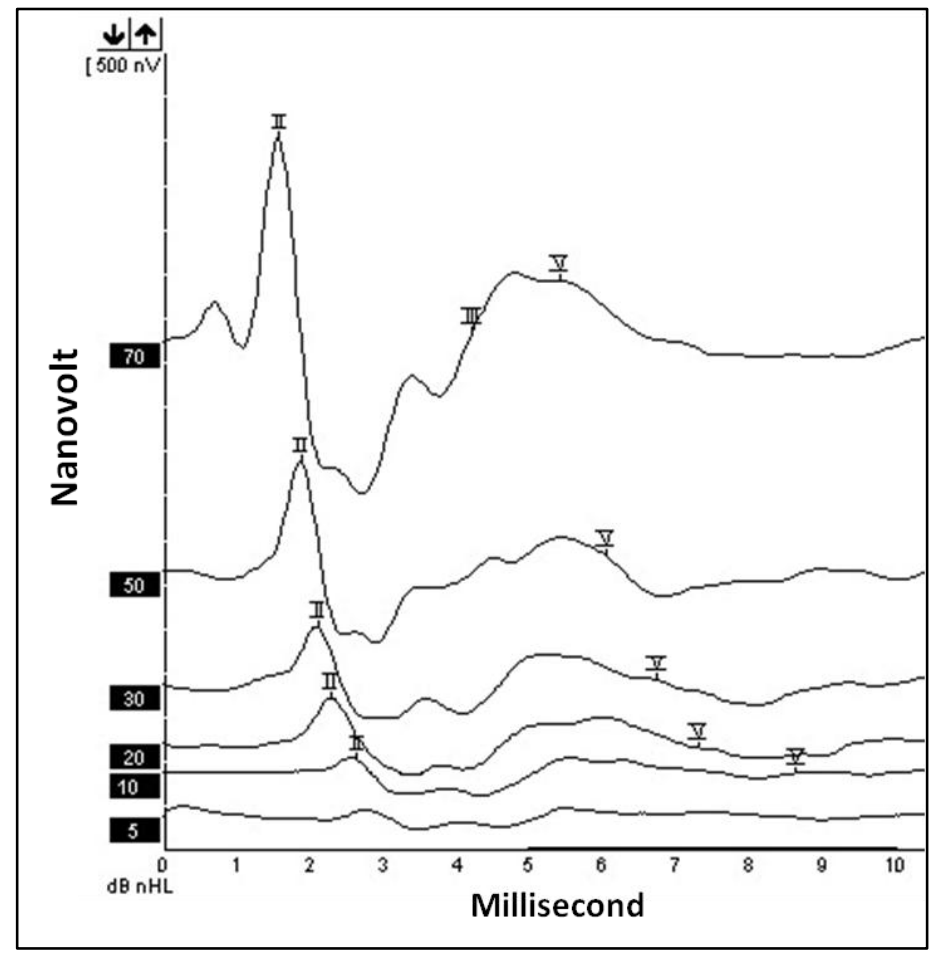

Figure 3: An output from the auditory brainstem responses (ABR) threshold determination

\section{Results}

Mean ABR threshold shift values (the difference between the baseline and 4 weeks post-exposure are depicted in figure 4 . The results revealed that exposure to noise produced a significant ABR threshold shift in the noise-exposed group ranging from $9.88 \mathrm{~dB}$ at $4 \mathrm{kHz}$ to $6.11 \mathrm{~dB}$ at $16 \mathrm{KHz}$.

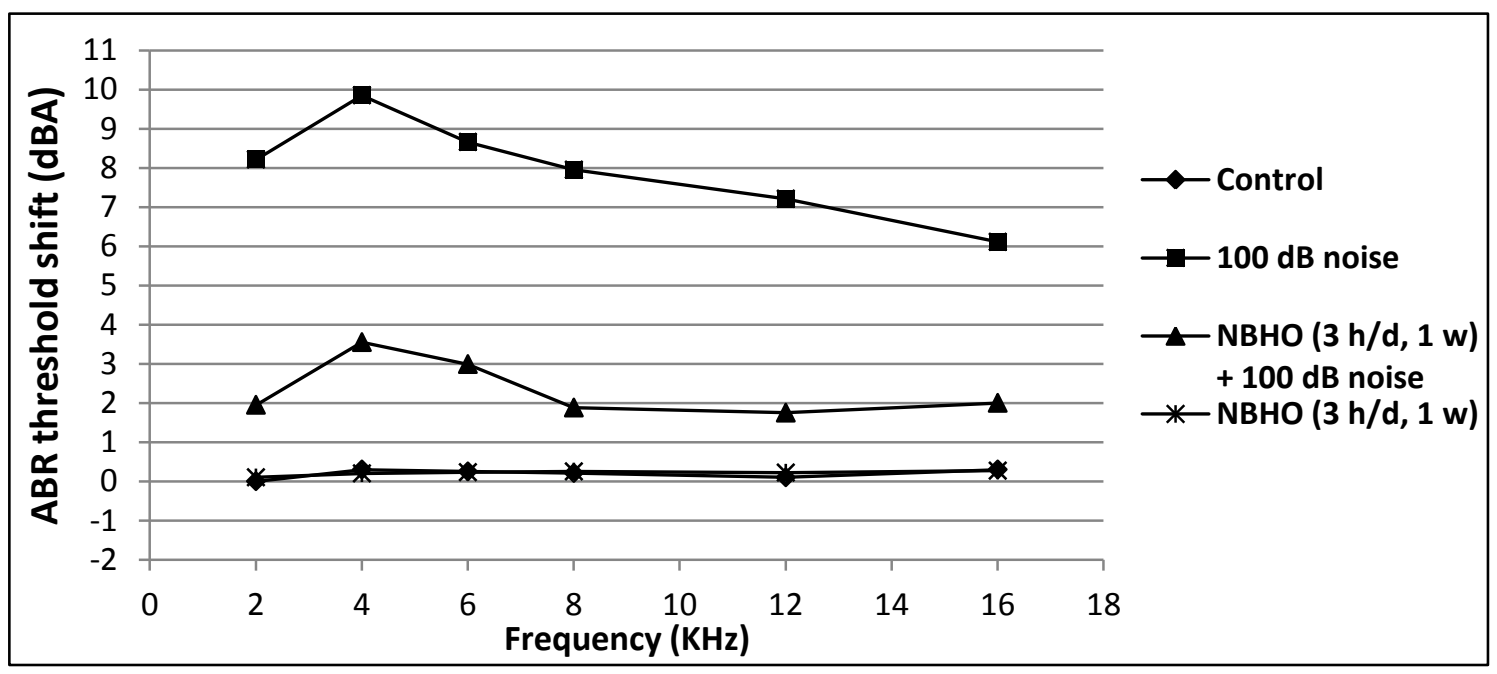

Figure 4: Mean auditory brainstem responses (ABR) threshold shift values in all experimental groups ABR: Auditory brainstem responses; NBHO: Normobaric hyperoxia 


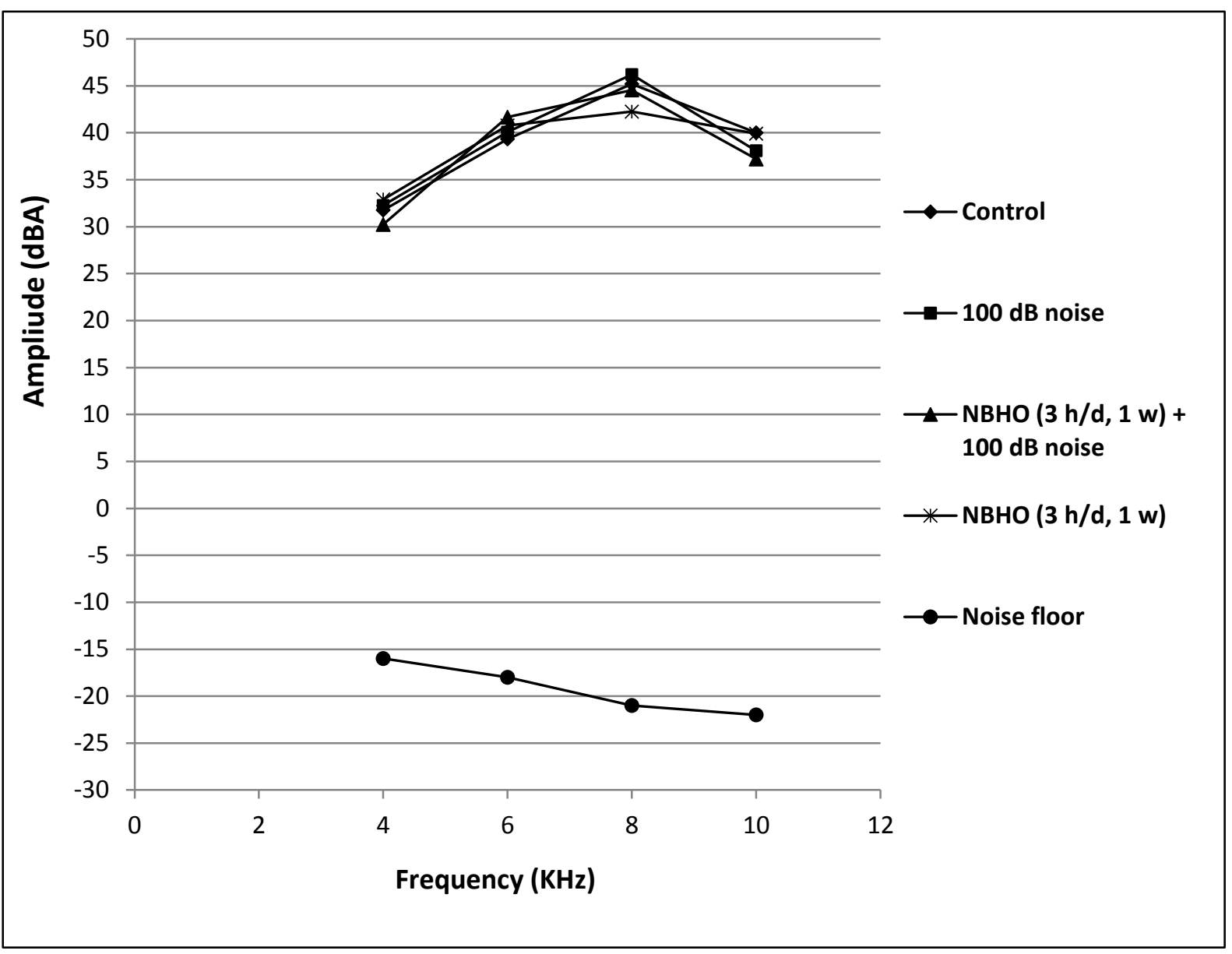

Figure 5: Mean values of baseline distortion product otoacoustic emissions (DPOAE) amplitudes [signal to noise ratio (SNR)] as a function of $\mathrm{f}_{2}$ frequency in all experimental groups

NBHO: Normobaric hyperoxia

The subjects from the NBHO (3 hours/day, 1 week) $+100 \mathrm{~dB}$ group also showed threshold shift values. ANOVA and Tukey's tests showed that the subject in NBHO (3 hours/day, 1 week) $+100 \mathrm{~dB}$ noise had significantly elevated threshold shift values compared to the subjects from the control and the groups that were only preconditioned by NBHO (no exposure to noise) at all tested frequencies and click ABR $(\mathrm{P}<0.001)$. Nonetheless, when the results are compared to the noise-exposed subjects, the rats that had received normobaric hyperoxia, came up with reduced levels of click and tone burst hearing threshold shift values at all frequencies. Figures 5 and 6 show the mean values of preexposure and 4 weeks post-exposure DPOAE amplitudes at $\mathrm{f}_{2}$ frequencies of $4,6,8$ and 10 $\mathrm{kHz}$ in all experimental groups. The DPOAE audiograms showed an almost similar level of amplitudes before exposure to noise. The results obtained by DPOAE recordings revealed that exposure to a noise level of 100 $\mathrm{dB}$ for 6 hours/day, 5 days/week within 4 weeks, contributed to a significant decrease in DPOAE amplitudes at all tested frequencies ( $\mathrm{P}$ $<0.001$ ).

The statistical analysis also showed that the mean values of DPOAE amplitudes obtained from the NBHO (3 hours/day, 1 week) +100 $\mathrm{dB}$ noise were significantly higher than the noise-exposed group, emphasizing the role of oxygen preconditioning to reduce the level of NIHL. 


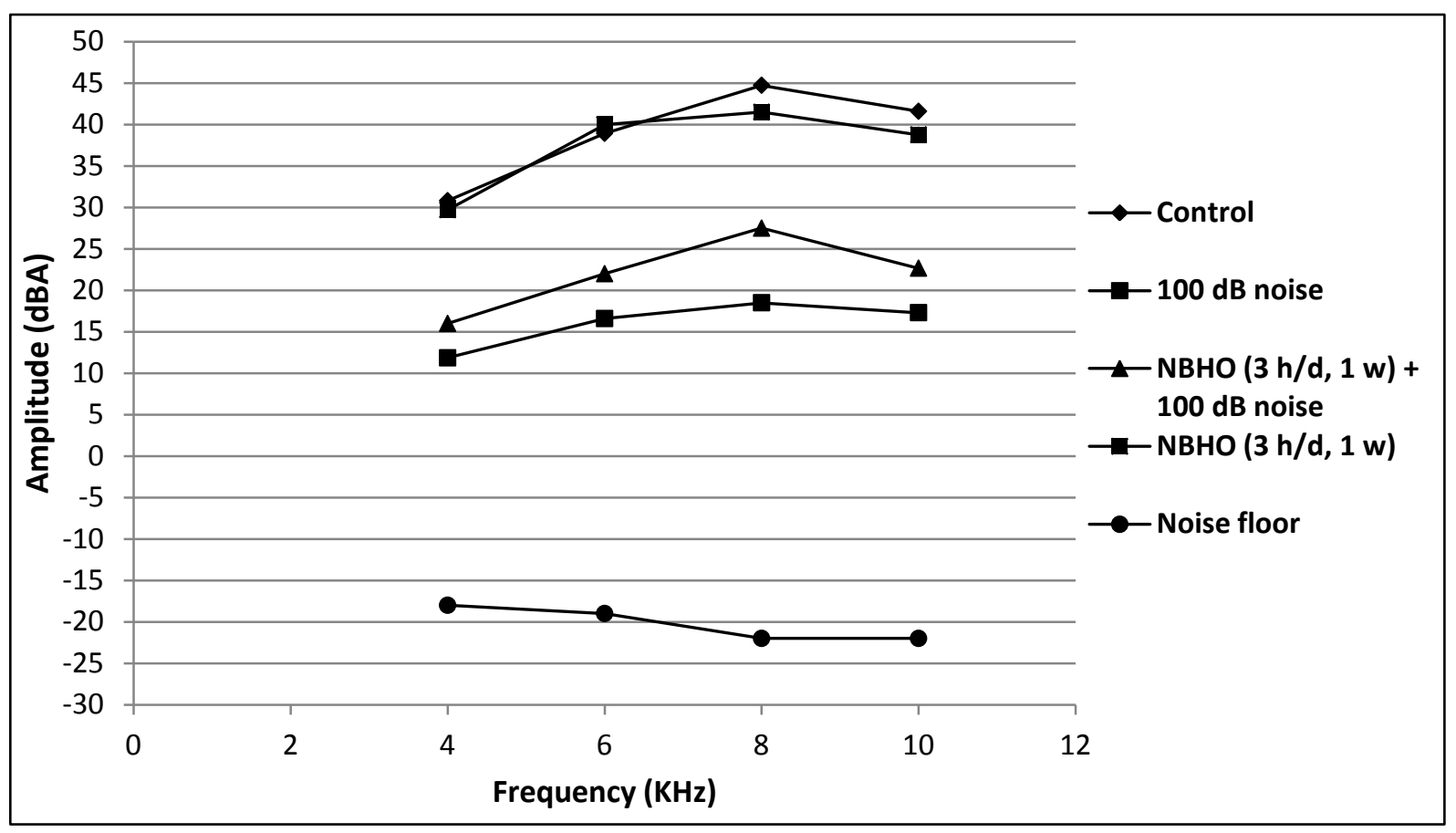

Figure 6: Mean values of distortion product otoacoustic emissions (DPOAE) amplitudes [signal to noise ratio $(\mathrm{SNR})] 4$ weeks post-exposure as a function of $\mathrm{f}_{2}$ frequency in all experiment groups

NBHO: Normobaric hyperoxia

\section{Discussion}

The inhalation of NBHO improved the ABR and DPOAE recordings of the noise-exposed group.

The literature review reveals that the preconditioning with oxygen particularly by NBHO has a significant protective role in developing ischemic tolerance and improving the cerebral blood flow and oxygenation (9, 10, 20). It is now evident that applying NBHO significantly protects the subjects against the development of several disorders and is a low cost and non-invasive method of conditioning (21). It is well documented that inhalation of NBHO maximizes the level of oxygen dissolution in the blood and enhances the bond between hemoglobin and oxygen (17).

Exposure to the higher levels of noise also results in the reduction of cochlear blood flow and lack of proper cellular oxygenation, and in turn, disrupts the cellular metabolism and homeostasis due to the formation of free radicals. This might consequently contribute to the malfunction of the cochlea in general and its OHCs in particular (22).

The results from the present study indicated that the weekly preconditioning of the rats by NBHO resulted in improved levels of ABR threshold shifts and DPOAE amplitudes when compared to the controls.

In spite of the fact that we did not aim to investigate any possible underlying mechanism for oxygen preconditioning, several studies have indicated that the NBHO preconditioning usually induces ischemic tolerance as well as enhancing protective enzymatic activities (23). NBHO preconditioning is also the main contributor to increasing antioxidant enzymes such as superoxide dismutase, catalase, glutathione peroxidase and glutathione reductase activities in the cochlea (6).

It is worth noting that other preconditioning stressors such as noise (conditioning and toughening) (24), heat (25) and restraint (26) seem to follow the same mechanism and exert 
their protective effect via elevated levels of antioxidant enzymes in the cochlea.

Although there is evidence for the effectiveness of the NBHO, the time window and duration for its application as a therapeutic or preventive agent is still controversial. While Rasoulian et al. reported that the weekly preconditioning by pure oxygen initiates several protective mechanisms in the rat's body $(27,28)$, other researchers emphatically have mentioned the role of prolonged preconditioning by oxygen. For instance, Joglekar et al. indicated that 30-minute inhalation of normobaric pure oxygen before exposure to a noise level of $100 \mathrm{~dB}$ (SPL) at $1000 \mathrm{~Hz}$ is an advantageous protocol in terms of both the amount of threshold shifts and recovery rates (29). The inhalation of $100 \%$ normobaric oxygen by guinea pigs during the exposure to $105 \mathrm{~dB}$ broadband noise for 6 hours/day for 5 consecutive days decreased noise-induced threshold shifts. The authors reported that cochlear oxygenation effectively protects the cochlea against NIHL and emphasized the role of duration of inhalation as a critical factor for the conditioning process and protection (30).

In the present study, the groups of rats that received NBHO preconditioning almost experienced a sub-lethal oxidative preconditioning that leads to a mild oxidative stress. This may effectively stimulate endogenous defense mechanisms such as antioxidant molecules and enzymes against ROS formation and injury, and hence provided reasonable levels of protection (31). Therefore, the protective effects are remarkably evident in their electrophysiological test results.

Moreover, according to the previous studies, the intermittent inhalation of hyperbaric oxygen ( 1 hour/day for 5 days) has resulted in higher activity of antioxidant enzymes such as catalase and superoxide dismutase in the spinal cord tissue of the rabbits (32). Also, there is well-documented evidence about the fact that di-methylthiourea, a scavenger of oxygen-free radicals, disrupted the ischemic tolerance induced by hyperoxic condition (33).
The previous studies suggest that administration of NBHO to the patients during anesthesia and surgery is a safe procedure (34) particularly if inhaled for less than 6 hours (35) and the NBHO might begin to affect the rats' lungs only after 40 hours of continuous exposure (36). Nonetheless the possible side effects of lengthy oxygenation and breathing pure oxygen should also be taken into account in the future studies as well.

\section{Conclusion}

The results from our study indicated that the weekly inhalation of $\mathrm{NBHO}$ by the rats that were not exposed to the noise did not show any significant levels of hearing loss. It seems that still further work is required to investigate the multiple aspects of NBHO preconditioning and its preventive effects on NIHL development and hearing impairment. It is therefore recommended that future studies consider the chronic aspects of hyperoxia preconditioning and noise exposure in order to have a more realistic outcome.

\section{Acknowledgements}

This study was supported by Tarbiat Modares University (2012-2016). The authors would like to particularly thank Doctor Mehdi Akbari and Doctor Fatemeh Heidari, the two audiologists, who provided insight and expertise that greatly assisted us in the implementation of DPOAE and ABR tests.

Conflict of Interest: None declared

\section{Refrences}

1. Nelson DI, Nelson RY, Concha-Barrientos M, Fingerhut M. The global burden of occupational noise-induced hearing loss. Am J Ind Med 2005; 48(6):446-58.

2. Dobie RA. The burdens of age-related and occupational noise-induced hearing loss in the United States. Ear Hear 2008; 29(4):565-77.

3. Jafari MJ, Karimi A, Haghshenas M. Extrapolation of experimental field study to a national occupational noise exposure standard. International Journal of Occupational Hygiene 
2010; 2(2):63-8

4. Duan M, Qiu J, Laurell G, Olofsson A, Counter SA, Borg E. Dose and time-dependent protection of the antioxidant N-Lacetylcysteine against impulse noise trauma. Hear Res 2004; 192(1-2):1-9.

5. Poirrier AL, Pincemail J, Van Den Ackerveken P, Lefebvre PP, Malgrange B. Oxidative stress in the cochlea: an update. Curr Med Chem 2010; 17(30):3591-604.

6. Le Prell CG, Yamashita D, Minami SB, Yamasoba T, Miller JM. Mechanisms of noiseinduced hearing loss indicate multiple methods of prevention. Hear Res 2007; 226(1-2):22-43.

7. Yoshida N, Liberman MC. Sound conditioning reduces noise-induced permanent threshold shift in mice. Hear Res 2000; 148(1-2):213-9.

8. Nasrniya S, Bigdeli MR. Normobaric hyperoxia (HO) preconditioning induces durable and effective neuroprotection against cerebral ischemia and mGluRII expression. Biomedicine \& Aging Pathology 2013; 3(3):137-44.

9. Bigdeli MR, Hajizadeh S, Froozandeh M, Rasulian B, Heidarianpour A, Khoshbaten A. Prolonged and intermittent normobaric hyperoxia induce different degrees of ischemic tolerance in rat brain tissue. Brain Res 2007; 1152:228-33.

10. Bigdeli MR. Preconditioning with prolonged normobaric hyperoxia induces ischemic tolerance partly by upregulation of antioxidant enzymes in rat brain tissue. Brain Res 2009; 1260:47-54.

11. Mohammadi E, Bigdeli MR. Effects of preconditioning with normobaric hyperoxia on $\mathrm{Na}^{+} / \mathrm{Ca}^{2+}$ exchanger in the rat brain. Neuroscience 2013; 237:277-84.

12. World Medical Association. World Medical Association Declaration of Helsinki. Ethical principles for medical research involving human subjects. Bull World Health Organ 2001; 79(4):373-4.

13. Chen G, Decker B, Krishnan Muthaiah VP, Sheppard A, Salvi R. Prolonged noise exposure-induced auditory threshold shifts in rats. Hear Res 2014; 317:1-8.

14. Lataye R, Campo P, Loquet G. Combined effects of noise and styrene exposure on hearing function in the rat. Hear Res 2000; 139(1-2):86-96.

15. Lataye R, Campo P, Loquet G, Morel G. Combined effects of noise and styrene on hearing: comparison between active and sedentary rats. Noise Health 2005; 7(27):49-64.

16. Hinners RG, Burkart JK, Punte CL. Animal inhalation exposure chambers. Archives of Environmental Health: An International Journal
1968; 16(2):194-206.

17. Khvoles R, Freeman S, Sohmer H. Effect of temperature on the transient evoked and distortion product otoacoustic emissions in rats. Audiol Neurotol 1998; 3(6):349-60.

18. Popelar J, Groh D, Pelnov J, Canlon B, Syka J. Age-related changes in cochlear and brainstem auditory functions in Fischer 344 rats. Neurobiol Aging 2006; 27(3):490-500.

19. Alvarado JC, Fuentes-Santamara V, JarenoFlores T, Blanco JL, Juiz JM. Normal variations in the morphology of auditory brainstem response (ABR) waveforms: a study in wistar rats. Neurosci Res 2012; 73(4):30211.

20. Shin HK, Dunn AK, Jones PB, Boas DA, Lo EH, Moskowitz MA, et al. Normobaric hyperoxia improves cerebral blood flow and oxygenation, and inhibits peri-infarct depolarizations in experimental focal ischaemia. Brain 2007; 130(Pt 6):1631-42.

21. Chen C, Cui H, Li Z, Wang R, Zhou C. Normobaric oxygen for cerebral ischemic injury. Neural Regen Res 2013; 8(31):2885-94.

22. Lamm K, Arnold W. Successful treatment of noise-induced cochlear ischemia, hypoxia, and hearing loss. Ann N Y Acad Sci 1999; 884:233-48.

23. Lamm K, Lamm C, Arnold W. Effect of isobaric oxygen versus hyperbaric oxygen on the normal and noise-damaged hypoxic and ischemic guinea pig inner ear. Adv Otorhinolaryngol 1998; 54:59-85.

24. Alvarado JC, Fuentes-Santamaría V, GabaldónUll MC, Jareño-Flores T, Miller JM, Juiz JM. Noise-induced "Toughening" effect in wistar rats: enhanced auditory brainstem responses are related to calretinin and nitric oxide synthase upregulation. Front Neuroanat 2016; 10:19.

25. Yoshida N, Kristiansen A, Liberman MC. Heat stress and protection from permanent acoustic injury in mice. J Neurosci 1999; 19(22):1011624.

26. Wang Y, Liberman MC. Restraint stress and protection from acoustic injury in mice. Hear Res 2002; 165(1-2):96-102.

27. Rasoulian B, Jafari M, Mahbod M, Dehaj ME, Nowrozi M, Wahhabaghai $\mathrm{H}$, et al. Pretreatment with oxygen protects rat kidney from cisplatin nephrotoxicity. Ren Fail 2010; 32(2):234-42.

28. Rasoulian B, Mohammadhosseniakbari H, Kadkhodaee M, Mofid M, Baqeri Gh, Bigdeli $\mathrm{MR}$, et al. Preconditioning with oxygen attenuates rat renal ischemia-reperfusion injury. J Surg Res 2008; 146(2):282-8.

29. Joglekar SS, Lipscomb DM, Shambaugh GE Jr. Effects of oxygen inhalation on noise-induced 
threshold shifts in humans and chinchillas. Arch Otolaryngol 1977; 103(10):574-8.

30. Hatch M, Tsai M, LaRouere MJ, Nuttall AL, Miller JM. The effects of Carbogen, carbon dioxide, and oxygen on noise-induced hearing loss. Hear Res 1991; 56(1-2):265-72.

31. Arslan HH, Satar B, Serdar MA, Ozler M, Yilmaz E. Effects of hyperbaric oxygen and dexamethasone on proinflammatory cytokines of rat cochlea in noise-induced hearing loss. Otol Neurotol 2012; 33(9):1672-8.

32. Nie H, Xiong L, Lao N, Chen S, Xu N, Zhu Z. Hyperbaric oxygen preconditioning induces tolerance against spinal cord ischemia by upregulation of antioxidant enzymes in rabbits. J Cereb Blood Flow Metab 2006; 26(5):666-74.

33. Zhang X, Xiong L, Hu W, Zheng Y, Zhu Z, Liu Y, et al. Preconditioning with prolonged oxygen exposure induces ischemic tolerance in the brain via oxygen free radical formation. Can J Anaesth 2004; 51(3):258-63.

34. Kabon B, Kurz A. Optimal perioperative oxygen administration. Curr Opin Anaesthesiol 2006; 19(1):11-8.

35. Tinits P. Oxygen therapy and oxygen toxicity. Ann Emerg Med 1983; 12(5):321-8.

36. Stogner SW, Payne DK. Oxygen Toxicity. Ann Pharmacother 1992; 26(12):1554-62. 